\title{
Study of low-energy internal Bremsstrahlung radiation from ${ }^{147} \mathrm{Pm}$
}

\author{
M.K. Raghavendra*, C.R. Ramaswamy \\ Department of Physics, Bangalore University, Jnana Bharathi Campus, Bangalore-560056, India
}

Received 6 August 2003; accepted 3 March 2004

\begin{abstract}
The absolute intensity of the internal Bremsstrahlung radiation following the beta decay of ${ }^{147} \mathrm{Pm}$ has been measured using a high-resolution X-ray detector in the low-energy region from 3 to $33 \mathrm{keV}$. Magnetic-deflector method has been used to avoid the betas from reaching the detector. The corrected spectral intensity has been compared with the Nilsson-corrected KUB theory. Good agreement between experiment and theory is seen in this energy region.

(C) 2004 Elsevier Ltd. All rights reserved.
\end{abstract}

Keywords: Internal Bremsstrahlung; Beta decay; X-rays; Absolute intensity; Detour transition

\section{Introduction}

Internal Bremsstrahlung (IB) is a weak and continuous electromagnetic spectrum accompanying the beta decay and Electron Capture (EC) processes. There has been a number of studies on IB, both experimental $(\mathrm{Sr}$. Little Flower et al., 1990; Basha et al., 1991; Norman et al., 1991; Budick et al., 1992; Khalil, 1993; Khalil and Basha, 1995) and theoretical (Keshava et al., 1997; Frolov and Smith, 1998). A wide discrepancy between theoretical predictions and the experimental IB spectra has been reported for many radioisotopes (Basavaraju et al., 1983; Gunes and Basar, 1992; Sr. Little flower et al., 1992). The disagreement has, in general, been attributed mainly to poor quality of experimental data and non-inclusion of Coulomb, relativistic and detour effects in the theory (Badiger and Umakantha, 1989). Even with the inclusion of these effects by Nilsson (1956), Lewis and Ford (1957) and others the disagreement persists; the disagreement appears to be greater in the case of forbidden transitions. However, in the lowerenergy region, below $35 \mathrm{keV}$, these effects (except that of

*Corresponding author. Tel.: +91-80-2321-4001 × 332; fax: +91-80-2321-9295.

E-mail address: maigurer@hotmail.com (M.K. Raghavendra). the Coulomb effect) are noted to be small. Hence, comparison between experiment and theory would be straightforward in this energy region. It is interesting to note that in a few cases, where a high-resolution detector has been used, the agreement is found to be good (Badiger and Umakantha, 1989; Sr. Little flower et al., 1990; Moriyama and Minowa, 1995).

The beta decay of ${ }^{147} \mathrm{Pm}$ to ${ }^{147} \mathrm{Sm}$ occurs dominantly to the ground state $(99.994 \%$ ) (Lederer and Shirley, 1978) (inset of Fig. 2). This process is classified as a nonunique first forbidden transition. The earlier work of Prasad et al. (1974) on ${ }^{147} \mathrm{Pm}$ reports good agreement with the predictions of the theory of Lewis and Ford (1957); conversely, the more recent work of Babu et al. (1985) reports wide disagreement with all the IB theories. In both studies, comparison of the IB spectrum has been performed only in the higher-energy region (above $35 \mathrm{keV}$ ) and the data were obtained using a poor resolution $\mathrm{NaI}(\mathrm{Tl})$ detector. Keeping this in mind, the IB spectrum from ${ }^{147} \mathrm{Pm}$ has been investigated for the first time using a high-resolution Si-PIN diode detector in the low-energy region $(3-33 \mathrm{keV})$, in the present work.

\section{Experimental technique}

A carrier-free ${ }^{147} \mathrm{Pm}$ radioactive source in the form of promethium chloride in dilute $\mathrm{HCl}$ was obtained from 
Board of Radiation and Isotope Technology, Mumbai (India). The solution was evaporated drop-by-drop on a thin mylar sheet of thickness $25 \mu \mathrm{m}$ to form an active area of $5 \mathrm{~mm}$ diameter. The source strength was $2.775 \mathrm{MBq}$.

The IB spectrum was measured using a high-resolution ( $300 \mathrm{eV}$ at $6.4 \mathrm{keV}$, with a $12 \mu$ s shaping constant from the amplifier) Si-PIN diode detector (XR100 T, Amptek Inc., USA). The magnetic-deflection method was used in order to prevent beta particles from entering the detector. A schematic of the experimental arrangement is shown in Fig. 1. Details of the experimental setup are given elsewhere (Raghavendra and Ramaswamy, 2001).

The detector output was suitably shaped and amplified using a spectroscopic amplifier (Canberra Inc., USA). The amplified pulse was recorded using a $2 \mathrm{~K}$ -

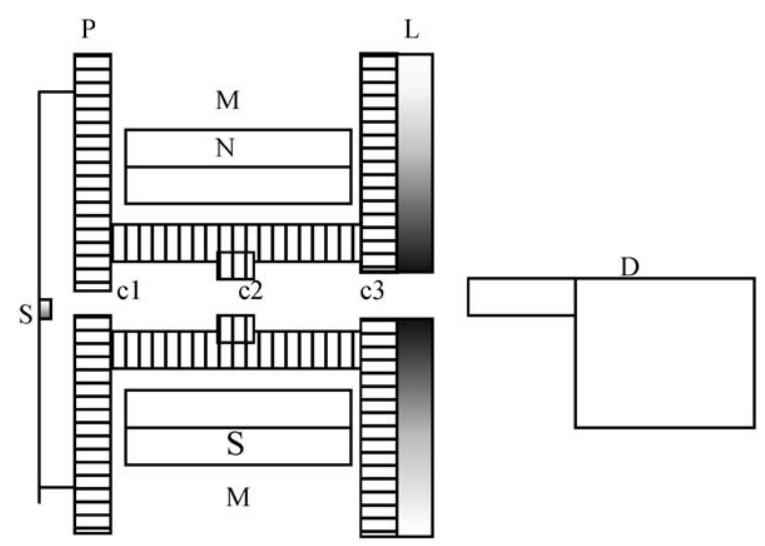

Fig. 1. Schematic experimental arrangement (not to scale). S: source, D: Si-PIN diode detector, c1,c2,c3 : Collimators, P: Perspex housing, L : Lead shield with aluminum lining, M: Permanent magnets.

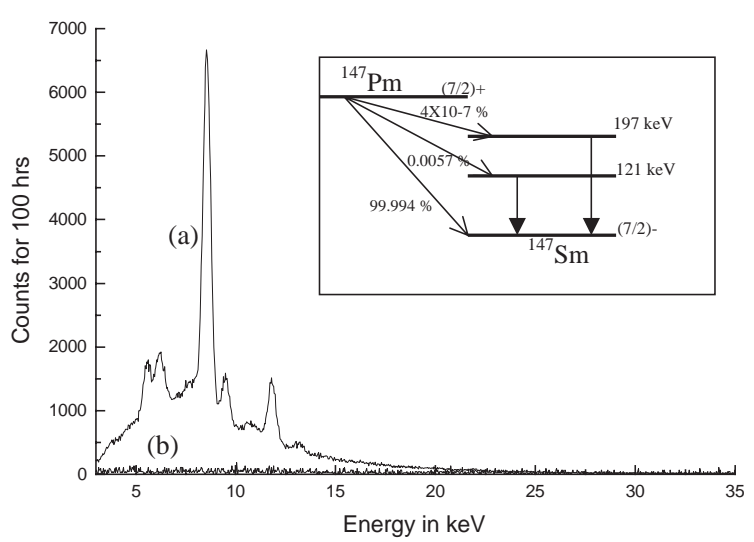

Fig. 2. (a) Raw IB spectrum from ${ }^{147} \mathrm{Pm}$; (b) background spectrum. Inset: decay scheme of ${ }^{147} \mathrm{Pm}$ (Lederer and Shirley, 1978).
ADC/MCA attached to a personal computer. Energy calibration was done using standard X-ray sources. The IB data and background data from 3 to $33 \mathrm{keV}$ were collected alternately for a duration of $120 \mathrm{~h}$ each, in cycles of $10 \mathrm{~h}$. The raw and background spectra are shown in Fig. 2.

\section{Data analysis and results}

The raw spectrum, given in Fig. 2, shows a continuous spectrum on which resides a series of X-ray peaks. These peaks were identified to be the L-X-ray peaks of Sm (5.65 and $6.21 \mathrm{keV}), \mathrm{K}-\mathrm{X}$-ray peaks of $\mathrm{Zn}(8.56 \& 9.52)$ (Lederer and Shirley, 1978) and a sum peak of emissions at 5.65 and $6.21 \mathrm{keV}$ (namely $11.86 \mathrm{keV}$ ). The L-X-ray peaks are due to internal conversion of the weak $121 \mathrm{keV}$ level of ${ }^{147} \mathrm{Sm} . \mathrm{Zn}$ is a probable contaminant of reactorbased sources such as ${ }^{147} \mathrm{Pm}$.

The background-subtracted spectrum has been corrected for detector resolution and detector efficiency. Correction for energy resolution becomes essential since a mono-energetic gamma line spreads into a Gaussian distribution due to statistical fluctuations. The FWHM was experimentally determined for photo peaks obtained from a set of standard X-ray sources and a best fit to these values was used to obtain FWHM at other energies.

The intrinsic detector efficiency for X-rays below $10 \mathrm{keV}$ is known to be $100 \%$ but practically it suffers from a low transmission in the beryllium window of the detector. Conversely, X-rays above $10 \mathrm{keV}$ have nearly $100 \%$ transmission in the beryllium window but suffer from low intrinsic-detector efficiency. As such, X-rays below $10 \mathrm{keV}$ were corrected for transmission loss and those above $10 \mathrm{keV}$ for detector efficiency. The absolute IB intensity per $\mathrm{keV}$ per beta decay was estimated from the final corrected spectrum using knowledge of the absolute source strength and the source-detector geometry.

In addition, the data have been corrected for air absorption effect. Mass attenuation coefficient for air (with composition of $78 \% \quad \mathrm{~N}_{2}$ and $22 \% \mathrm{O}_{2}$ ) was estimated at standard energies from 3 to $33 \mathrm{keV}$ using the XCOM program (Berger and Hubbell, 1991, Version 3.1). The ratio of intensity transmitted through $11 \mathrm{~cm}$ of the air (the air path used in present investigation), to the incident intensity $\left(I / I_{0}\right)$ calculated from these values were used for air-absorption correction.

The spectrum shown in Fig. 3 represents a smoothly varying IB continuum on which resides a set of characteristic radiations described earlier. Using a multi-peak fit analysis (PeakFit Ver 4.1 from Systat Inc., USA), the intensities of the characteristic peaks were estimated and subtracted. The resulting base-line fit to the data represents the true IB continuum. The absolute IB intensity $/ \mathrm{keV} /$ beta decay has been compared 


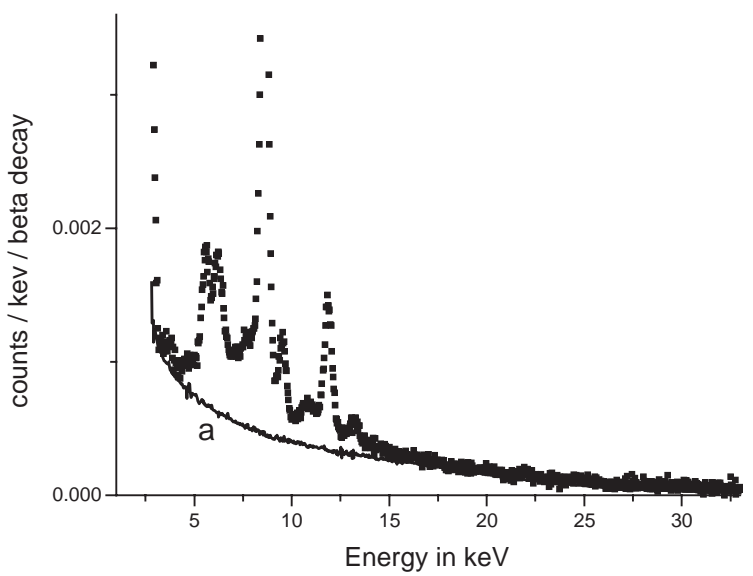

Fig. 3. The corrected IB spectrum with L-X-ray peaks of ${ }^{147} \mathrm{Sm}$. The Solid-line (a) is the base-line fit to the IB data obtained using a multi-peak fit program (PeakFit Ver 4.1 from Systat Inc., USA).

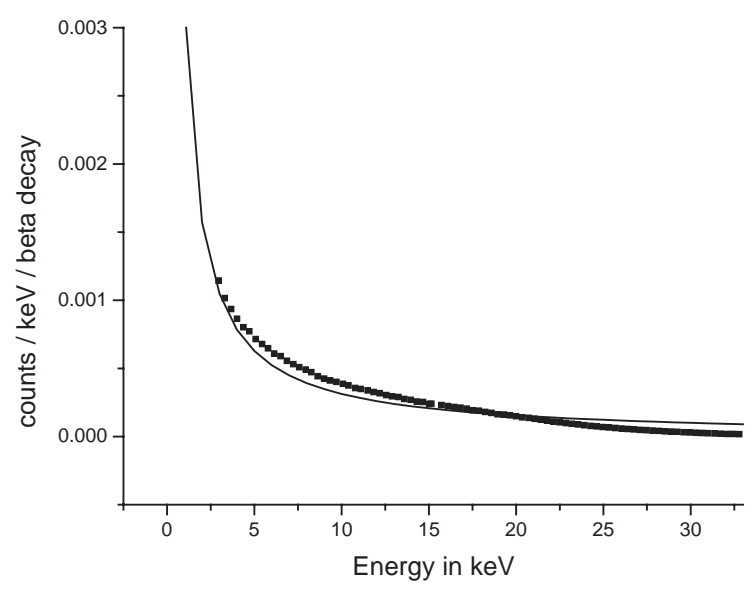

Fig. 4. Comparison of absolute intensity of IB spectrum (squares) with the theoretical prediction from Nilsson-corrected KUB theory (solid line). The normalization factor used was 1.07. Statistical errors are less than the size of the points.

with the theoretical predictions of the Nilsson-corrected KUB theory (with a normalization factor of 1.07). This is shown in Fig. 4.

Fig. 4 shows overall good agreement (to within 3.8\%) between the measured intensity and the theoretical predictions, in the energy region from 3 to $33 \mathrm{keV}$. The disagreement of $3.8 \%$ in the total intensity may be expected due to the presence of characteristic peaks in the experimental spectrum. Overall agreement in the low-energy region below $35 \mathrm{keV}$ thus confirms the validity of the Nilsson-corrected KUB theory. This also suggests that detour and relativistic effects are indeed not important in the low-energy region even in the case of a forbidden transition. A similar result was obtained in our earlier work on ${ }^{204} \mathrm{Tl}$ (Raghavendra and Ramaswamy, 2001).

\section{Acknowledgements}

This experiment has been carried out using the Research Grant received from the Department of Atomic Energy (India).

\section{References}

Babu, B.R.S., Basavaraju, A., Venkataramaiah, P., Gopala, K., Sanjeeviah, H., 1985. Inner Brremsstrahlung accompanying beta decay of ${ }^{147} \mathrm{Pm}$. Phys. Rev. C 32, 1010-1013.

Badiger, N.M., Umakantha, N., 1989. Internal Bremsstrahlung spectrum of ${ }^{35} \mathrm{~S}$ in the range from 10 to $100 \mathrm{kev}$. Phys. Rev. C 39, 216-218.

Basavaraju, A., Venkataramaiah, P., Gopala, K., Sanjeviah, H., 1983. Internal Bremsstrahlung accompanying beta decay in ${ }^{90} \mathrm{Sr}-{ }^{90} \mathrm{Y}$. Phys. Rev. C 28, 333-336.

Basha, A.M., Khalil, E.I., Hussein, M., Ragab, H.S., El-Konsol, 1991. Inner Bremsstrahlung accompanying non-unique II forbidden beta decay of ${ }^{36} \mathrm{Cl}$. Z. Phys. A $338,3-6$.

Budick, B., Chen, J., Lin, H., 1992. Inner Bremsstrahlung from tritium beta decay. Phys. Rev. C 46, 1267-1275.

Frolov, A.M., Smith, V.H., 1998. Inner Bremsstrahlung radiation emitted during beta decay and related problems. Phys. Rev. A 58, 1212-1220.

Gunes, T., Basar, S., 1992. Internal Bremsstrahlung from ${ }^{204} \mathrm{Tl}$. Acta. Phys. Hungarica 71, 157-160.

Keshava, S.L., Venkataramaiah, P., Gopal, K., Gopinath, D.V., 1997. Exact Coulomb corrections in beta decay and inner Bremsstrahlung. Indian J. Phys. 71A, 481-491.

Khalil, E.I., 1993. Internal Bremsstrahlung accompanying nonunique I forbidden beta decay of ${ }^{141} \mathrm{Ce}$. Z. Naturforschung. 48A, 1115-1118.

Khalil, E.I., Basha, A.M., 1995. Investigation of the influence of nuclear charge on the Internal Bremsstrahlung accompanying the beta decay of ${ }^{45} \mathrm{Ca}$ and ${ }^{141} \mathrm{Ce}$. Indian J. Phys. A 69A, 351-359.

Lederer, C.M., Shirley, V.S., 1978. Table of Isotopes 7th Edition). Wiley, New York pp. 802.

Lewis Jr., R.R., Ford, G.W., 1957. Coulomb effects in Internal Bremsstrahlung. Phys. Rev. 107, 756-765.

Moriyama, S., Minowa, M., 1995. The $17 \mathrm{kev}$ neutrino and the search for anomalous $\gamma$ rays in ${ }^{35} \mathrm{~S}$ decay. Phys. Lett. B 347, $152-157$.

Nilsson, S.B., 1956. On the Coloumb effect for the Internal Bremsstrahlung accompanying beta decay. Ark. Fys. 10, 467-477.

Norman, E.B., Sur, B., Lesko, K.T., Hindi, M.M., Larimer, R.M., Ho, T.R., Witort, J.T., Luke, P.N., Hansen, W.L., Haller, E.E., 1991. Evidence for the emission of a massive 
neutrino in nuclear beta decay. J. Phys. (London) G 17, S291-S299.

Prasad, B.R., Narashimhamurthy, V.A., Narashimhamurthy, K., 1974. Internal Bremsstrahlung from ${ }^{147} \mathrm{Pm}$. J. Phys. A 7, 2295-2303.

Raghavendra, M.K., Ramaswamy, C.R., 2001. Internal Bremsstrahlung spectrum of ${ }^{204} \mathrm{Tl}$ in the X-ray region. Int. J. Mod. Phys. E 10, 237-243.
Sr. Little Flower, Babu, B.R.S., Mukerjee, R.N., Baliga, B.B., 1990. Internal Bremsstrahlung from ${ }^{63} \mathrm{Ni}$. J. Phys. (London) G 16, 1873-1879.

Sr. Little Flower, Mukerjee, R.N., Babu, B.R.S., Indira, A., Venkataramaiah, P., Gopala, K., Neelakandan, K., Baliga, B.B., 1992. Internal Bremsstrahlung from ${ }^{45} \mathrm{Ca}$ and ${ }^{91} \mathrm{Yr}$. Indian J. Phys. 66A, 343-353. 